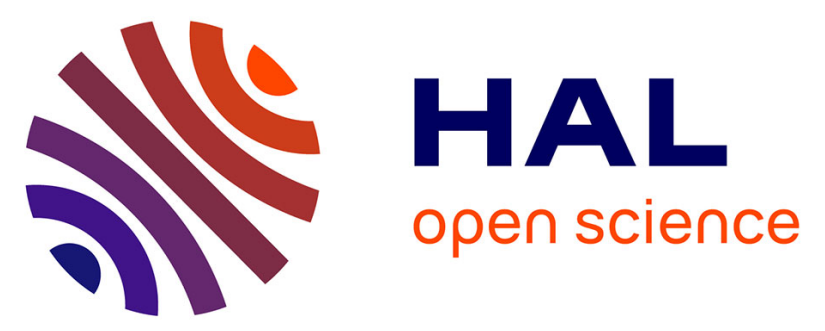

\title{
Comprehensive characterization of interface and oxide states in metal/oxide/semiconductor capacitors by pulsed mode capacitance and differential isothermal capacitance spectroscopy
}

Pierre R. Muret

\section{To cite this version:}

Pierre R. Muret. Comprehensive characterization of interface and oxide states in metal/oxide/semiconductor capacitors by pulsed mode capacitance and differential isothermal capacitance spectroscopy. Journal of Vacuum Science and Technology, 2014, 32 (3), pp.03D114. 10.1116/1.4865912 . hal-00965450v2

HAL Id: hal-00965450

https://hal.science/hal-00965450v2

Submitted on 21 Sep 2015

HAL is a multi-disciplinary open access archive for the deposit and dissemination of scientific research documents, whether they are published or not. The documents may come from teaching and research institutions in France or abroad, or from public or private research centers.
L'archive ouverte pluridisciplinaire HAL, est destinée au dépôt et à la diffusion de documents scientifiques de niveau recherche, publiés ou non, émanant des établissements d'enseignement et de recherche français ou étrangers, des laboratoires publics ou privés. 


\title{
Comprehensive characterization of interface and oxide states in metal/oxide/semiconductor capacitors by pulsed mode capacitance and differential isothermal capacitance spectroscopy
}

\author{
P. Muret* \\ Univ. Grenoble Alpes, Inst. NEEL, \\ F-38042 Grenoble, France \\ CNRS, Inst NEEL, F-38042 Grenoble, France
}

(Dated: March 25, 2014)

\begin{abstract}
In metal/insulator/semi-conductor structures, capacitance-voltage characteristics and capacitance or voltage transients can be measured in different conditions, which are described and implemented. Each method contains information about charges which are accommodated, captured or emitted by energy levels or bands at interface and inside the oxide. Pulsed capacitance measurements and differential isothermal procedures are analysed and performed. Calibration of the energy scale from the interface potential as a function of the applied voltage and extraction of the interface state spectra and characteristic response times independently are possible with the help of signal processing by Fourier transform of transients at one or few selected temperatures. Different trap filling conditions may help to discriminate between interface and oxide states. These methods are applied to $\mathrm{Al} / \mathrm{SrTiO}_{3} / \mathrm{Si}$ capacitors as an example.
\end{abstract}

PACS numbers: 73.40.Qv, 84.37.+q

\section{INTRODUCTION}

With the advent of insulating stacks using compound high-k dielectrics on silicon and other semiconductors, accurate characterization of defect states in these structures is increasingly needed. Extensive characterization of interface in metal/insulator/semiconductor (MIS) capacitors by electrical measurements under both steady-state bias voltage and transient regime $[1,2]$ have been performed since the sixties, stimulated initially by the development of metal $/ \mathrm{SiO}_{2} / \mathrm{Si}$ structures then more generally in metal/insulator/semiconductor devices. Because capacitance measurements under pulsed mode and in transient regime afford more versatile and powerful control of the carrier populations, methods relying on deep level transient spectroscopy (DLTS), either at constant voltage or constant capacitance (CC-DLTS), became increasingly used $[1,3,4]$. However, the Fermi level position at interface, or equivalently the energy scale at dielectricsemiconductor interface, was either deduced assuming the unsatisfactory hypothesis of a constant capture cross section of majority carriers by interface states or derived from an inaccurate method involving double filling pulses and the search for a quantity related to the capture cross section. Moreover, standard DLTS and CC-DLTS were firstly unable to discriminate between different families of interface and insulator states characterized by different time constant at the same temperature because of a single time window for the analysis of the transient and secondly the temperature scan used in DLTS induced variations of many physical quantities simultaneously, making model adjustment more difficult. Conversely, isothermal transient spectroscopy (ITS) [5] is free of these drawbacks and able to solve in a very smart and direct way the calibration of the energy scale at interface provided it is used in differential mode [6]. But the original analog ITS technique relying on the product of time and derivative of the capacitance or its square suffered from a non optimal signal over noise ratio $(\mathrm{S} / \mathrm{N})$ and poor resolution in the frequency domain. Fast Fourier transform (FFT) of the digitized capacitance transient [7] is now able to yield both much better $\mathrm{S} / \mathrm{N}$ because the calculation uses the whole transient, and precise time constant for the excess carriers population relaxation, which can be accurately extracted from several Fourier coefficient spectra in the frequency domain. In the following, isothermal pulsed capacitance and transient methods are analysed with the help of calculations which are fully detailed in the Appendix and applied to $\mathrm{Al} / \mathrm{SrTiO} 3 / \mathrm{Si}$ capacitors.

\section{PULSED CAPACITANCE METHOD}

All measurements are performed in a PhysTech FT1030 spectrometer which uses a Boonton 72B capacitance meter, whose analog output signals are digitized, and FFT processing of the transient signals. Firstly, the pulsed capacitance method is presented and used in a $\mathrm{Al} / \mathrm{SrTiO} 3 / \mathrm{p}-\mathrm{Si} / \mathrm{p}^{+}-\mathrm{Si}$ capacitor provided with a $50 \mathrm{~nm}$ insulating layer [8], annealed $30 \mathrm{~min}$. at $450{ }^{\circ} \mathrm{C}$ under $\mathrm{H}_{2}$ atmosphere. The silicon layer is doped with $2 \times 10^{15} \mathrm{~cm}^{-3}$ boron atoms and epitaxially grown on degenerate substrate. The high frequency capacitance $C_{t}\left(V_{G, i}\right)$ of the MIS structure is measured after the end of an accumulation or flat band pulse at each step of the gate voltage $V_{G, i}$ with a variable delay (Fig. 1). Interface states are full of majority carriers up to the energy level reached during this pulse and the charge 


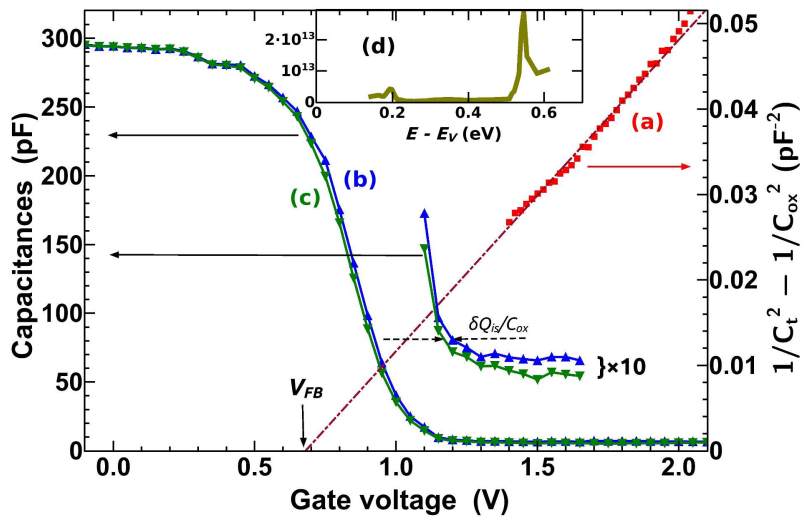

FIG. 1. (Color Online) (a) Difference of the inverse square capacitances of the MIS structure and the oxide layer measured after a $V_{F B}=0.68 \mathrm{~V}$ pulse within a delay of $d 0=200 \mu \mathrm{s}$; (b) MIS capacitance measured after a $-1 \mathrm{~V}$ accumulation pulse within a delay of $200 \mu \mathrm{s}$; (c) MIS capacitance measured after a $-1 \mathrm{~V}$ accumulation pulse within a delay of $d 1=2 \mathrm{~s}$; (d) interface state density $\left(\mathrm{eV}^{-1} \cdot \mathrm{cm}^{-2}\right)$ deduced from the voltage shift between (b) and (c) curves. Measurement temperature is $250 \mathrm{~K}$, low enough to inhibit inversion charge building.

trapped in them changes negligibly at least in the interface energy range above the Fermi level at flat bands in a p-type semiconductor after the response time of the set-up, which is $200 \mu \mathrm{s}(d 0)$. Thus, if the pulse voltage is chosen at the flat band voltage $V_{F B}$ after some trials, $V_{F B}$ can be deduced rigorously from the data from $\left(C_{t}^{H F, d 0}\right)^{-2}-\left(C_{o x}\right)^{-2}=\frac{2}{q \varepsilon_{S C} N_{d i}}\left(V_{F B}-V_{G}\right)$ (equation A.11) in the depletion and deep depletion regimes, as shown in Fig. 1 (a). If the pulse voltage induces accumulation, the same measurement method can be used with two different delays ( $d 0$ and $d 1$ ) (Fig. 1 (b) and (c) respectively). The first one $(d 0)$ is the same as previously and the second one $(d 1)$ is such that interface states has enough time to re-emit their trapped carrier and become empty. From the gate voltage difference $V_{G}^{d 1}-V_{G}^{d 0}=\frac{\left.Q_{i s}\right\rfloor_{\phi s}^{\phi_{s 0}}}{C_{o x}}=\frac{Q_{i s}\left(\phi_{s 0}\right)-Q_{i s}\left(\phi_{s}\right)}{C_{o x}}$ (equation A.13), the interface charge is calculated. Temperature is low enough (here $250 \mathrm{~K}$ ) to keep the MIS free of inversion charge up to delay $d 1$ which is here $2 \mathrm{~s}$. Finally, the ratio of the increment in this interface charge and that of the surface potential $\delta \phi_{s}=\frac{C_{o x}-C_{t}}{C_{o x}}\left[\delta V_{G}+\frac{\delta Q_{i s}^{\infty}}{C_{o x}}\right]$ (equation A.17) yields the interface density of states $N_{i s}$ in Fig. 1 (d) as a function of the energy $E$ at interface assessed from $V_{F B}$ and equation A.17 applied step by step. Although this method is not accurate when $N_{i s}$ is smaller than a few $10^{12} \mathrm{eV}^{-1} \cdot \mathrm{cm}^{-2}$, a peak in $N_{i s}$ is found near midgap like in similar samples already studied [9]. Methods using differential capacitance spectroscopy are more sensitive and additionally bring informations about the transient dynamics as discussed in the next section.

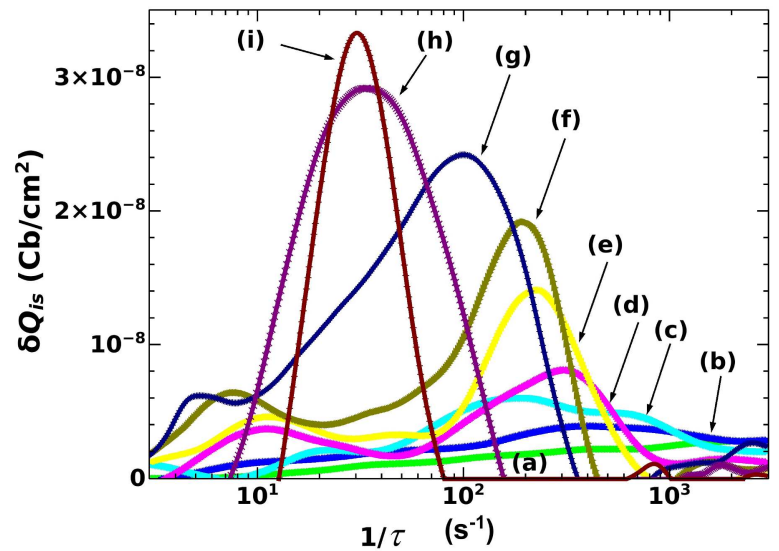

FIG. 2. (Color Online) (a) Differential isothermal spectra calculated from the first real coefficient of the FFT of voltage transients at $V_{G}=V_{F B}(\mathrm{a})+0.13 \mathrm{~V} ;$ (b) $+0.18 \mathrm{~V}$; (c) $+0.23 \mathrm{~V}$; (d) +0.28 V; (e) +0.33 V; (f) +0.38 V; (g) +0.43 V; (h) $+0.48 \mathrm{~V}$; (i) $+0.53 \mathrm{~V}$; with $V_{F B}=-0.67 \mathrm{~V}$ and a temperature of $260 \mathrm{~K}$ in the p-type MIS capacitor. Position of the maxima yields the inverse time constant $e_{p}^{\prime}$ of each transient. Two main families can be seen in the spectra, corresponding to two different capture cross sections.

\section{DIFFERENTIAL ISOTHERMAL TRANSIENT SPECTROSCOPY}

A much more sensitive method consists in performing the difference between two transient regimes that have been recorded at two gate voltage values $V_{G, i}$ and $V_{G, i+1}$ after a voltage pulse $V_{p}$ affording the same carrier concentration at interface with the same duration $t_{p}$. These regimes can be either the capacitance at constant gate voltages $V_{G, i}$ and $V_{G, i+1}$ or the voltage at constant capacitances $C_{t}\left(V_{G, i}\right)$ and $C_{t}\left(V_{G, i+1}\right)$, both lasting a time window $t_{w}$. Measurements are repeated as a function of $t_{w}$ in order to get spectra from the FFT, at various $V_{G, i}$ (Fig. 2) in samples which are prepared in the same way as previously, except that the oxide thickness is now 15 $\mathrm{nm}$, the MIS structures underwent no post-annealing and were achieved on both p- and n-type silicon.

In order to exemplify the two methods, differential ITS at constant capacitance (CC-ITS) and $\partial Q_{i s}=-C_{o x} \partial V_{G}^{L F}$ (equation A.12) are used for the p-type MIS while constant voltage ITS and $\delta Q_{i s}=$ $q N_{D} \varepsilon_{S C} C_{o x}\left[\frac{\partial C_{t, i}}{C_{t, i}^{3}}-\frac{\partial C_{t, i-1}}{C_{t, i-1}^{3}}\right]$ (equation A.16) are used for the n-type MIS. Peaks position in the different spectra, as one can see in Fig. 2, allows to derive the inverse response time, or equivalently the inverse time constant $e_{p}^{\prime}$ (or $e_{n}^{\prime}$ ) of each interface state family. Only the main amplitude is considered in the following.

After performing the same calculations as in the previous section to get the surface potential, $N_{i s}$ can be derived (Fig. $3(\mathrm{a})$ ) and the capture cross section $\sigma_{p}$ can be de- 


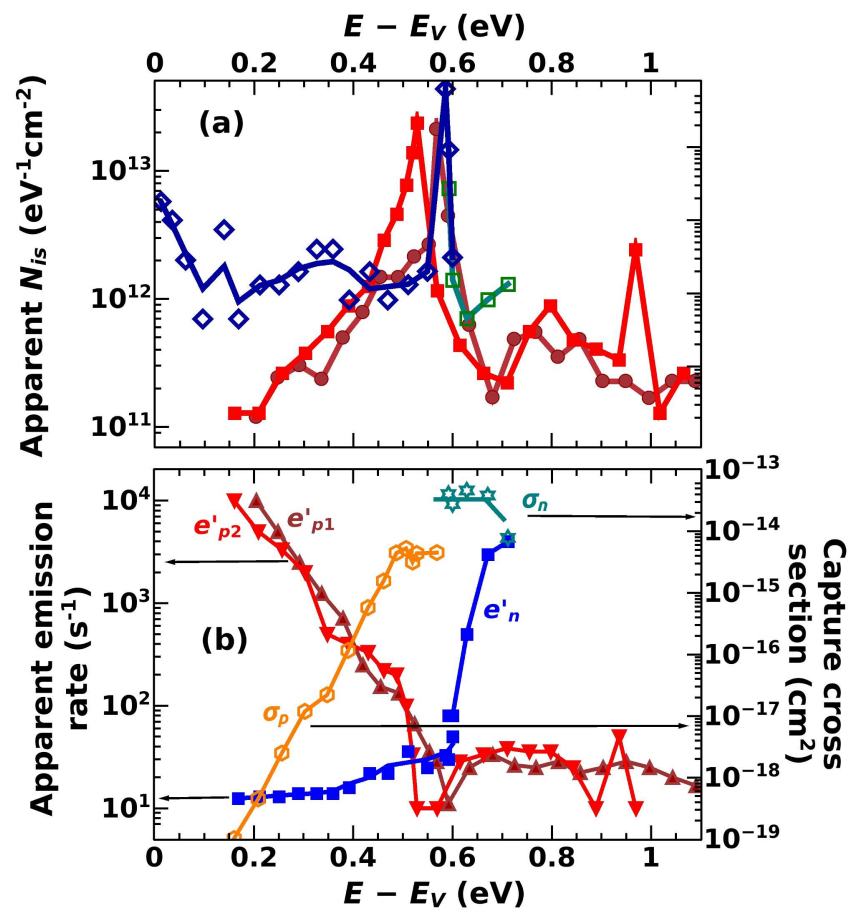

FIG. 3. (Color Online) (a) Apparent interface states density in full symbols for the p-type MIS capacitor (circles for $t_{p}=1 \mu \mathrm{s}$ and squares for $t_{p}=1 \mathrm{~ms}$ ) deduced from differential CC-ITS at $260 \mathrm{~K}$ and open symbols for the n-type MIS capacitor from differential CC-ITS at 250K; (b) Reciprocal time constants of the transients $e_{p 1}^{\prime}$ for $t_{p}=1 \mu \mathrm{s}, e_{p 2}^{\prime}$ and $e_{n}^{\prime}$ for $t_{p}=1 \mathrm{~ms}$; and capture cross sections of the interface states for majority carriers when the derivation is reliable. In these MIS structures, flat band voltages are $-0.67 \mathrm{~V}$ and $+0.79 \mathrm{~V}$ for p-type and n-type MIS respectively, as derived from the pulse capacitance method described in the previous section.

duced from the emission rate $e_{p}=\sigma_{p} v_{t h p} N_{V} \exp \left[\left(E_{V}-\right.\right.$ $E) / k T]$ for p-type when it dominates the reciprocal time constant $1 / \tau=e_{p}^{\prime}$ (Fig. $3(\mathrm{~b})$ ), with $v_{t h p}$ the thermal velocity of holes and $N_{V}$ the effective density of states at the valence band edge $E_{V}$, or symmetrically for the n-type MIS. It must be stressed that the interface state density obtained in the p-type MIS with two values of the filling duration $t_{p}$ and that derived for the n-type MIS are very similar near midgap, with a prominent peak between $E_{V}+0.53 \mathrm{eV}$ and $E_{V}+0.58 \mathrm{eV}$. A second peak appears for the longer filling pulse duration $t_{p}=1 \mathrm{~ms}$, probably related to an oxide state because all the proper interface states are already filled at the shorter $t_{p}=1 \mu \mathrm{s}$, the capture time constant being well below $1 \mu$ s in accumulation regime. The presence of a second peak nearer the conduction band is confirmed qualitatively by the standard inverse square capacitance characteristics of the n-type MIS capacitor, which shows two inflexions (not shown) because of the term $\left.Q_{i s}\right|_{0} ^{\phi_{s}} / C_{O x}$ which has to be substracted from $V_{F B}$ in A.11. Above the main peak, the energy range of $N_{i s}$ derived from ITS at constant voltage in the n-type MIS capacitor is limited to only $E_{V}+0.71 \mathrm{eV}$ because of the failure of the depletion approximation beyond this limit. Conversely, $N_{i s}$ can be evaluated closer to the valence band edge in the p-type MIS capacitor with the CC-ITS method, not limited to the depletion regime. However, due to the emission rate of majority carriers which becomes much larger than the set-up limit, the density of states is notably underestimated in comparison to that deduced in the n-type MIS capacitor where the emission rate is much lower in this energy range, well below the conduction band edge. The reciprocal time constants $e_{n}^{\prime}$ and $e_{p}^{\prime}$ shown in Fig. 3 (b) should display a "U" curve if they were equal to the sum of the emission rates of majority and minority carriers $e_{n}+e_{p}$, minority carrier capture being negligible due to the low temperature which prevents the inversion charge to have time to develop. But only the branch which increases toward the majority carriers band edge appear in each type. On the contrary, near midgap and toward the minority carriers band edge, $e_{n}^{\prime}$ and $e_{p}^{\prime}$ become constant. Such an effect could be an indication of the participation of charge migration inside the oxide because the term $\partial \bar{Q}_{o x}^{L F}$ which has to be added to $\partial Q_{i s}^{L F}$ in equation A.5 is proportional to the product of the total charge within the insulator by the ratio of the barycentre of this charge to the insulator thickness, but not likely dependent on the surface potential. Outside this energy range, capture cross sections can be firmly deduced (Fig. 3 (b)). Other ITS spectra would be necessary at lower temperatures to study all the previous physical quantities in the energy ranges closer to the band edges.

\section{CONCLUSION}

The methods useful for interface states and oxide states characterization has been thoroughly analysed and exemplified with two kinds of $\mathrm{Al} / \mathrm{SrTiO}_{3} / \mathrm{Si}$ capacitors. Pulsed mode capacitance measurements allows for a rigorous derivation of the flat band voltage and net impurity concentration but lack of sensitivity for interface state density assessment. Differential FFT-processed ITS, eventually at several temperatures, fulfils all the needs for accurate evaluations.

\section{CHARGES, VOLTAGES AND CAPACITANCES IN A MIS STRUCTURE}

\section{Basic equations}

In the MIS capacitance, relying on potential continuity, the potential voltage applied on the metal gate $V_{G}$ can be related to the potential at the insulator-semiconductor interface $\phi_{s}$, referred to as the surface potential in the following, and to the voltage difference across the insulator 
$V_{o x}$ through :

$$
V_{G}=\Phi_{M S}+V_{o x}+\phi_{s}
$$

where $\Phi_{M S}=\Phi_{M}-\chi-E_{G} / 2 e-\phi_{F}, \Phi_{M}$ being the metal work function, $\chi$ the electron affinity of the semiconductor, $E_{G}$ the band gap of the semiconductor, $\phi_{F}=$ $\left(E_{i}-E_{F}\right) / e$ the Fermi potential deduced from the difference between intrinsic and Fermi energy levels in the bulk semiconductor, $\phi_{s}$ the surface potential and $e$ the absolute electron charge. The electrical potential reference is taken in the neutral zone of the semiconductor as usual. Using the insulator capacitance $C_{o x}$ per unit surface and applying Gauss theorem from the neutral zone of the semiconductor to the insulator, $V_{o x}=$ $-\left[Q_{S C}+Q_{i s}+\overline{Q_{o x}}\right] / C_{o x}$, where all the charges are per unit surface, $Q_{S C}$ being the total charge in the semiconductor space charge zone, $Q_{i s}$ the charge accommodated by interface states and $\overline{Q_{o x}}$ the product of the total charge within the insulator by the ratio of the barycentre of this charge to the insulator thickness. Then

$$
\left.V_{G}=V_{F B}-\frac{1}{C_{o x}}\left(Q_{S C}+Q_{i s}\right\rfloor_{0}^{\phi_{s}}\right)+\phi_{s}
$$

where the flat band voltage is defined when $\phi_{s}=0$ :

$$
V_{F B}=\Phi_{M S}-\left(Q_{i s}(0)+\overline{Q_{o x}}\right) / C_{o x}
$$

$Q_{i s}(0)$ being the absolute charge in interface states at $\phi_{s}=0$, and $\left.Q_{i s}\right\rfloor_{0}^{\phi_{s}}$ the net charge when the surface potential goes from 0 to $\phi_{s}$, equal to $Q_{i s}\left(\phi_{s}\right)-Q_{i s}(0)$. Because one needs to calculate capacitances, charge and potential variations are evaluated in the following.

\section{Charge and potential variations}

Differentiating equation A.2 on the one hand for slow $(\mathrm{LF})$ and on the other hand for fast variations ( $\mathrm{HF}$ ) yields respectively :

$$
\partial V_{G}^{H F}=-\frac{1}{C_{o x}} \partial Q_{S C}^{H F}+\partial \phi_{s}^{H F}
$$

and

$$
\partial V_{G}^{L F}=-\frac{1}{C_{o x}}\left(\partial Q_{S C}^{L F}+\partial Q_{i s}^{L F}\right)+\partial \phi_{s}^{L F}
$$

where it is assumed that the interface states do not follow the AC HF signal used for probing the capacitance of the MIS structure. The LF variations are also those which are probed in transient measurements like in DLTS and ITS. If variations in the oxide charge happen, a term $\partial \bar{Q}_{o x}^{L F}$ has to be added to $\partial Q_{i s}^{L F}$ because these charges play the same role in equation A.2. In the following, $\partial \bar{Q}_{o x}^{L F}$ will be considered as included into $\partial Q_{i s}^{L F}$ if necessary.
In the general case, the charge variations $\partial Q_{S C}^{L F}$ and $\partial Q_{S C}^{H F}$ are determined by the semiconductor capacitance $C_{S C}$, where $Q_{S C}$ is a complicated function $\lambda_{D}^{-1} f\left(e \phi_{s} / k T, e \phi_{F} / k T\right), k$ being the Boltzman constant, $T$ the absolute temperature and $\lambda_{D}$ the Debye length. However, $Q_{S C}$ is a monotonic function of $e \phi_{s} / k T$ and its derivative $C_{S C}$ is a univocal function of $e \phi_{s} / k T$ if the inversion regime is excluded, so that it is possible to get their variations from their differential. The surface potential being defined as the electrical potential at the insulator-semiconductor interface as compared to that in the neutral zone, the depletion charge $Q_{S C}$ is negative in a p-type semiconductor (respectively positive in a n-type one) when $\phi_{s}$ goes to positive values (respectively negative values in a n-type semiconductor), while $V_{G}$ follows the same direction as $\phi_{s}$. These trends being unchanged in other regimes, charge variations in the semiconductor can be defined as follows:

$$
\partial Q_{S C}^{L F, H F}=-C_{S C} \partial \phi_{s}^{L F, H F}
$$

while the capacitance change is :

$$
\partial C_{S C}^{L F, H F}=\left(\lambda_{D}^{-1} \frac{\partial^{2} f}{\partial \phi_{s}^{2}}\right) \partial \phi_{s}^{L F, H F}
$$

The series HF capacitance can now be defined from A.4, A.6 and the opposite charge variations on the metal electrode $\partial Q_{M}^{H F}=C_{t} \partial V_{G}^{H F}$, where $C_{t}$ is the total capacitance of the whole MIS structure:

$$
C_{t}^{-1}=C_{o x}^{-1}+C_{S C}^{-1}
$$

\section{Depletion and deep depletion regimes}

If the measurements are done at $\phi_{s}$ values restricted to the depletion and deep depletion regime, $C_{S C}$ can be replaced by the depletion capacitance per unit surface $C_{D}$. If measurements are performed within a time delay, short enough after the end of the accumulation regime and at a sufficiently low temperature, the inversion charge cannot develop at surface potentials able to induce inversion. As a consequence, the depletion approximation remains valid and the deep depletion regime occurs. If the net dopant impurity concentration $N_{d i}$ is assumed to be homogeneous, depletion approximation allows writing $Q_{S C}=q N_{d i} w$ and $C_{D}=\varepsilon_{S C} / w$ for a depletion zone width $w$, a semiconductor permittivity $\varepsilon_{S C}$, and with $q=+|e|$ for a n-type semiconductor whereas $q=-|e|$ for a p-type semiconductor. Then, the depletion charge itself and its variation can be respectively expressed as:

$$
Q_{S C}=\frac{q N_{d i} \varepsilon_{S C}}{C_{D}}
$$

and

$$
\partial Q_{S C}^{L F}=Q_{S C} \frac{\partial w}{w}=-Q_{S C} \frac{\partial C_{D}}{C_{D}}
$$


The depletion width and surface potential can also be expressed as $w=\sqrt{\frac{2 \varepsilon_{S C}\left|\phi_{s}\right|}{e N_{d i}}}$ and $\phi_{s}=-\frac{q N_{d i} \varepsilon_{S C}}{2 C_{D}^{2}}$. From this last expression, A.2, A.9, and A.8, calculation of the relationship between gate voltage and $\mathrm{HF}$ capacitances becomes possible in the depletion regime, provided the term $\left.Q_{i s}\right|_{0} ^{\phi_{s}}$ is cancelled in A.2. Such a case is achieved if the total capacitance $C_{t}$ is measured in pulsed mode after the end of a voltage pulse inducing nearly flat bands, within a delay time $d 0$ short enough to keep the interface charge at $Q_{i s}(0)$. In such a delay time, the term $\left.Q_{i s}\right\rfloor_{0}^{0}$ vanishes in A.2 and after some algebraic manipulations, one can derive:

$$
\left(C_{t}^{H F, d 0}\right)^{-2}-\left(C_{o x}\right)^{-2}=\frac{2}{q \varepsilon_{S C} N_{d i}}\left(V_{F B}-V_{G}\right)
$$

From this equation, the doping concentration and flat band voltage can be deduced from the linear part of $\left(C_{t}^{H F, d 0}\right)^{-2}-\left(C_{o x}\right)^{-2}$ in depletion and deep depletion regimes after two or three iterations to ensure convergence of $V_{F B}$ with the $V_{G}$ value used to induce flat bands. Conversely, if $C_{t}^{H F}$ is not measured just after flat band conditions but rather with enough delay so that equilibrium population in interface states is reached at each $V_{G}$ step, the term $\left.Q_{i s}\right\rfloor_{0}^{\phi_{s}} / C_{o x}$ must be substracted from $V_{F B}$ in A.11. Since it is varying with $V_{G}$, both $V_{F B}$ and $N_{d i}$ are affected by errors when this method is used, because the first member of A.11 would not only reflect the depletion zone variation but also the interface population variation.

\section{Voltage transients at constant capacitance}

Firstly, calculation of transient voltages which can be measured at constant total capacitance are detailed. In constant capacitance DLTS (CC-DLTS) and ITS (CCITS), the total HF capacitance is maintained constant after the filling pulse by a feedback loop so that $\partial \phi_{s}=0$ from equations A.8 and A.7 and $\partial Q_{S C}=0$ from equation A.6, both for $\mathrm{HF}$ and $\mathrm{LF}$ regimes because if fast variations are quenched, slow variations too. Therefore, equation A.5 leads simply to

$$
\partial Q_{i s}=-C_{o x} \partial V_{G}^{L F}
$$

Extraction of the interface charge variations is therefore straightforward in all regimes except inversion and can be monitored as a function of time.

Secondly, if only the whole change in interface charge is needed, a much simpler version of the constant capacitance method can be implemented. As previously, it needs the measurement on the one hand of the capacitance as soon as possible after an accumulation pulse within a delay $d 0$, when all the interface states are full of majority carriers up to $\phi_{s 0}$, and on the other hand after a time delay $d 1$ long enough for a complete discharge of interface states in equilibrium at $\phi_{s}$. If $V_{G}^{d 0}$ and $V_{G}^{d 1}$ are respectively the corresponding gate voltages, their difference, calculated from equation A.2, yields only the following term when performed at the same total HF capacitance, that is at the same $\phi_{s}$ and $Q_{S C}$, irrespective of the regime (accumulation, flat band, depletion or deep depletion), except at inversion:

$$
V_{G}^{d 1}-V_{G}^{d 0}=\frac{\left.Q_{i s}\right\rfloor_{\phi_{s}}^{\phi_{s 0}}}{C_{o x}}=\frac{Q_{i s}\left(\phi_{s 0}\right)-Q_{i s}\left(\phi_{s}\right)}{C_{o x}}
$$

Therefore, the product of $V_{G}^{d 1}-V_{G}^{d 0}$ by $C_{o x}$ is the integrated interface charge between $\phi_{s}$ and $\phi_{s 0}$, and can be plotted as a function of the gate voltage. It exactly follows the change in oxide potential difference when interface states are either full or empty. When the gate voltage drives the junction towards inversion regime, the inversion charge may be added to the interface charge if the delay is sufficient to build this inversion charge. To avoid such effect, a trade-off about the delay $d 1$ has to be determined from transient experiments. In wide band gap semiconductors, a large margin generally exists between majority carriers emission time and inversion time, which can be exceedingly longer in MIS capacitors, so that this problem is not raised.

\section{Capacitance transients at constant voltage}

Alternatively, in ITS or DLTS performed at constant gate voltage after a filling pulse, the total voltage variation reads $\partial V_{G}^{L F}=0$. Using A.6 and A.8 in A.5 leads to:

$$
\partial Q_{i s}^{L F}=-\partial Q_{S C}^{L F}\left(1+\frac{C_{o x}}{C_{S C}}\right)
$$

In depletion and deep depletion regimes, $C_{S C}$ becomes $C_{D}$ and equations A.9, A.10 and its differential $\partial C_{D}^{-1}=$ $\partial C_{t}^{-1}$ can be used to derive a practical expression relying on the total capacitance variations, which are measurable at high frequency:

$$
\partial Q_{i s}^{L F}=q N_{d i} \varepsilon_{S C} C_{o x} \frac{\partial C_{t}}{C_{t}^{3}}
$$

Proportionality of the interface state charge variation to the amplitude of the transient capacitance is still valid, but limited to the depletion and deep depletion regimes. It is sufficient to know the net dopant impurity concentration $N_{d i}$, the oxide capacitance $C_{o x}$ and to measure the full amplitude $\partial C_{t}$ of the total capacitance transient in a long enough time window, and $C_{t}$ itself, or equivalently the ITS spectrum. If the measurements are done at subsequent values of the gate voltage $V_{G}^{i}(i=1,2, \ldots)$, the increment in the interface charge can be calculated with $\delta Q_{i s}=\partial Q_{i s}^{L F, i}-\partial Q_{i s}^{L F, i-1}$ from equation A.15:

$$
\delta Q_{i s}=q N_{D} \varepsilon_{S C} C_{o x}\left[\frac{\partial C_{t, i}}{C_{t, i}^{3}}-\frac{\partial C_{t, i-1}}{C_{t, i-1}^{3}}\right]
$$


In practice, the denominators $C_{t, i}^{3}$ and $C_{t, i-1}^{3}$ being measured at each step, the terms within bracket can be directly derived from the whole amplitude of the differential ITS spectrum (DITS) also measured at each gate voltage. In constant voltage DLTS and ITS, interface states are detected because they are emitting majority carriers to the majority band, which have an opposite sign to the depletion charge. Such a fact induces an increase of the total capacitance until it reaches steady state because a fraction of these majority carriers contribute to reduce the space charge zone width in order that the overall dipolar momentum of the junction be maintained. However, when the junction is driven deeper and deeper towards depletion from the $i-1$ to the $i$ step, $C_{t}^{3}$ decreases appreciably, irrespective of the semiconductor type, so that the bracket content in equation A.16 is positive, involving a loss of holes $\left(\delta Q_{i s}<0\right)$ for a p-type MIS or a loss of electrons $\left(\delta Q_{i s}>0\right)$ for a n-type MIS. These rather tricky considerations do not apply to differential ITS at constant capacitance (CC-DITS), which turns out to be simpler at the expense of a more involved experimental set-up.

\section{Interface states and oxide states densities}

The surface potential increment $\delta \phi_{s}=\phi_{s}^{L F, i}-\phi_{s}^{L F, i-1}$ has to be related to the charge increment and gate voltage increment $\delta V_{G}=V_{G}^{L F, i}-V_{G}^{L F, i-1}$ to calculate the interface state density. Replacing differentials by increments in equations A.5, A.6 and using A.8, leads to:

$$
\delta \phi_{s}=\frac{C_{o x}-C_{t}}{C_{o x}}\left[\delta V_{G}+\frac{\delta Q_{i s}^{\infty}}{C_{o x}}\right]
$$

where $\delta Q_{i s}^{\infty}=\delta Q_{i s}$ if the transient has been monitored until steady state, ideally after an infinite time. In practice, either the time window $t_{w}$ used for transient measurements or the time delay $d 1$ must be sufficient to ensure that the steady state has been reached. Recalling the previous remark about $\delta Q_{i s}$, the two increments appearing in this last equation have opposite signs, with a resulting absolute variation $\left|\delta \phi_{s}\right|$ systematically smaller than $\left|\delta V_{G}\right|$, due to the contribution of a part of the applied voltage to the completion of the charge within interface states. In the constant capacitance method, the total capacitance is constant only during the transient, but not when the gate voltage is incremented from one step to the next one. Therefore, $\delta Q_{i s}^{\infty}$ has to be derived from the variation taking place in equation A.13 in order to calculate $\delta \phi_{s}$. The ratio of the effective charge variation to the energy change at the interface is easily deduced from either A.12 or A.16, and A.17. It matches the interface states density $N_{i s}$ only if the occupancy function $f_{i s}$ of interface states goes to either one or zero at the end of the time window $t_{w}$, which is also the transient duration. The first reason can cause underestimation of $N_{i s}$ if emission of minority carriers occurs when $\phi_{s}$ is such that the Fermi level at the interface is closer to the minority carrier band edge than the majority one. In such a case, it may happen that the final electronic occupancy function $f_{i s}\left(t_{w}\right)$ is either not zero (n-type MIS) or not unity (p-type MIS) and hence, the measured charge variation is only a fraction of that which would arise if the whole carrier charge trapped within interface states had been completely emitted toward the majority carrier band. If this fraction is not known, only the apparent charge density spectrum $\left|\delta Q_{i s} /\left(e \delta \phi_{s}\right)\right|$ can be plotted. A second reason of departure from the effective $N_{i s}$ lies in the possible superimposition of the oxide states response, more likely in high-k dielectrics or multi-layer insulators which may contain traps. It is possible to discriminate whether the oxide states contribute or not to $\delta Q_{i s}$ from considerations about the kinetics of charging and discharging states. The main method consists in studying the differential isothermal transient spectra as a function of the pulse amplitude or duration $t_{p}$ at flat band or under accumulation regime, which can strongly influence the charge trapped in oxide states because of tunnelling of carriers from interface states and charge migration inside the insulator. Finally, once the flat band voltage $V_{F B}$ is known, the origin of electronic energies at the interface, corresponding to $\phi_{s}=0$, can be determined from the Fermi level position $E_{F}$ in the bulk, relative to the majority band edge. Then, each subsequent increment $\delta \phi_{s}$, calculated from equation (A.17), is added to the previous one and serves to scale the electronic energy at interface. This is the way for plotting either the effective charge density or interface state spectrum in the MIS structure under study.

* pierre.muret@neel.cnrs.fr

[1] E. H. Nicollian and J. R. Brews, MOS physics and technology, John Wiley and Sons ed. (New York, 1982)

[2] M. J. Uren, J. H. Stathis and E. Cartier, J. Appl. Phys. 80, 3915 (1996)

[3] N. M. Johnson, J. Vac. Sci. Technol. 21, 303 (1982)

[4] M. Schulz, Surf. Science 132, 422 (1983)

[5] E. Yamaguchi, Jap. J. Appl. Phys. 21, 1628 (1982)

[6] J. Esteve, J. Samitier, H. Altelarrea, A. Herms, J.R. Morante, Appl. Surf. Science 30, 120 (1987)

[7] S. Weiss, R. Kassing, Solid St. Electron. 31, 1733 (1988)

[8] C. Dubourdieu, H. Roussel, C. Jimenez, M. Audier, J.P. Sénateur, S. Lhostis, L. Auvray, F. Ducroquet, B. J. Sullivan, P. K. Hurley, S. Rushworth, L. Hubert-Pfalzgraf, Mat. Sc. Engineering B 118, 105 (2005)

[9] P. Muret, L. Auvray, O. Salicio, C. Dubourdieu, Mat. Sc. Semicond. Proc. 9885 (2006) 